\section{Hemangioblastoma cerebeloso y trombocitopenia. Reporte de caso}

\author{
SANTIAGO PATIÑO G.
}

\section{Cerebellar hemangioblastoma and thrombocytopenia. Report of one case}

The association between vascular tumors and thrombocytopenia is rare. Kasabach-Merritt Syndrome is seen in childhood and is characterized by hemangiomas and thrombocytopenia. A 42 years-old man with a cerebellar hemangioblastoma and thrombocytopenia, admitted with a subarachnoid hemorrhage is reported. The patient was operated and required a splenectomy to manage the thrombocytopenia. After the splenectomy the patient developed a subdural hematoma that was operated. Despite the surgical treatment, the patient died.

(Rev Med Chile 2016; 144: 521-525)

Key words: Cerebellar Neoplasms; Hemangioblastoma; Kasabach-Merritt Syndrome; Subarachnoid Hemorrhage; Thrombocytopenia.

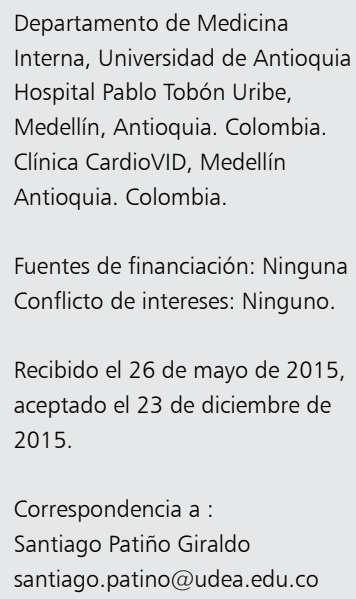

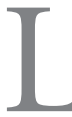
a cefalea es un motivo de consulta frecuente en la atención primaria y en medicina interna ${ }^{1}$. Los signos de alarma deben instar al clínico a la toma de neuroimágenes en búsqueda de causas pontencialmente fatales de la cefalea ${ }^{2}$. Los tumores cerebrales (benignos y malignos) explican $0,15 \%$ de las cefaleas y a su vez $78 \%$ de ellos tienen este síntoma ${ }^{3}$. Este artículo presenta un paciente con cuadro de cefalea con signos de alarma secundario hemangioblastoma cerebelar esporádico y trombocitopenia asociada, previamente descrito en 2 adultos como diagnóstico diferencial del Síndrome de Kasabach-Merrit (KMS).

\section{Caso clínico}

Hombre de 42 años de edad, agricultor, sin antecedentes de importancia, consulta en múltiples ocasiones por 3 meses de evolución de cefalea con sensación de pesadez y mareos sin respuesta a analgésicos comunes. En su evolución presenta ataxia y lateropulsión derecha. Una semana previo a su ingreso presenta endoforia derecha, diplopia y postración en cama por vértigo intenso. Se realiza TC simple de cráneo que demostró masa en fosa posterior y hemorragia subaracnoidea por lo que se traslada a hospital de nivel terciario. Ingresa al servicio de urgencias, consciente y orientado, con cefalea, diplopia e incapacidad para la marcha por vértigo. Su examen físico sólo demostró endoforia derecha, marcha inestable y lateropulsión derecha. Se realizó una RM simple y contrastada que reportó imagen compatible con hemangioblastoma (Figura 1). En exámenes de ingreso se evidenció recuento plaquetario de $10.000 / \mathrm{mL}$, sin otras alteraciones en serie roja ni blanca por lo que se difirió manejo quirúrgico y se iniciaron estudios complementarios. El perfil de autoinmunidad (ANAS, ENAS, Coombs, inmunoglobulinas, anticoagulante lúpico y anticardiolipinas) infeccioso (VIH, Hepatitis B y C), pruebas de coagulación (PT, PTT) así como los niveles de micronutrientes fueron normales. No se consideró medición de dímero D y fibrinógeno ante ausencia de síntomas sugerentes de coagulopatía.

El paciente presentó deterioro neurológico por lo que se decidió llevar a cirugía urgente previo soporte transfusional de plaquetas para resección de la masa en SNC. Por trepanación occipital derecha se pasa catéter de derivación ventricular externa al ventrículo lateral ipsilateral, con obtención de LCR a presión. Se identificó tumor en fosa posterior, 


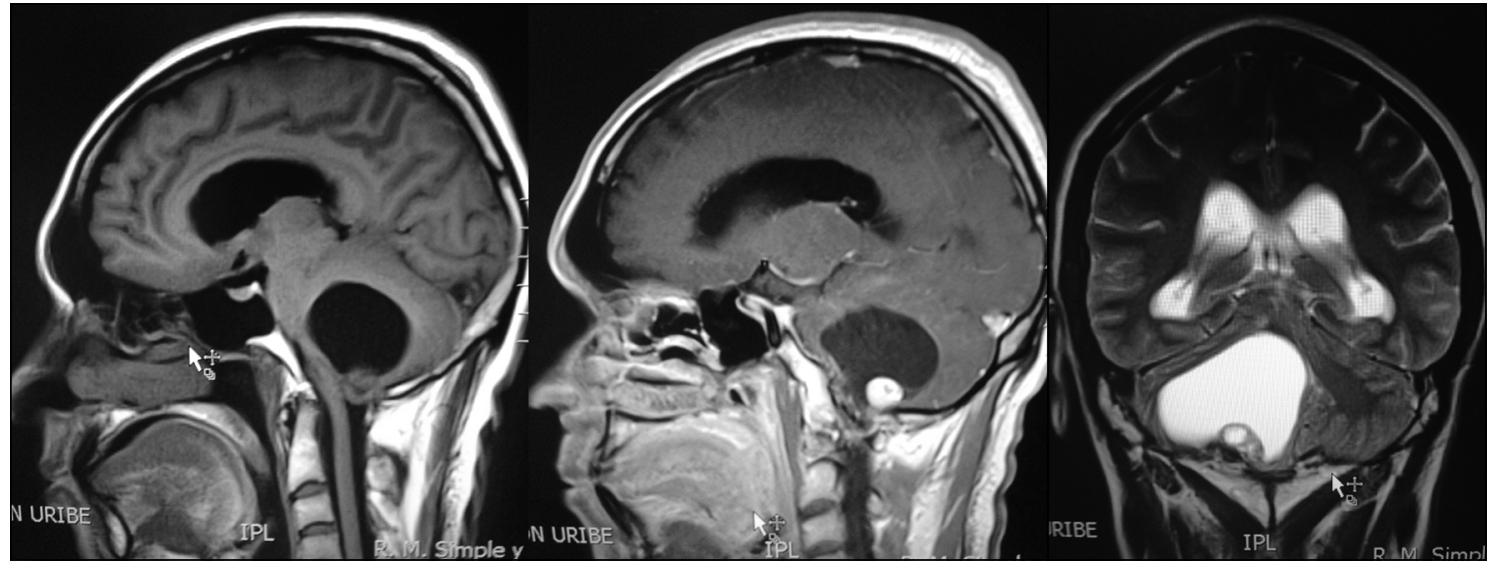

Figura 1. Lesión quística del hemisferio cerebeloso derecho con nódulo mural hipercaptante con gadolinio.

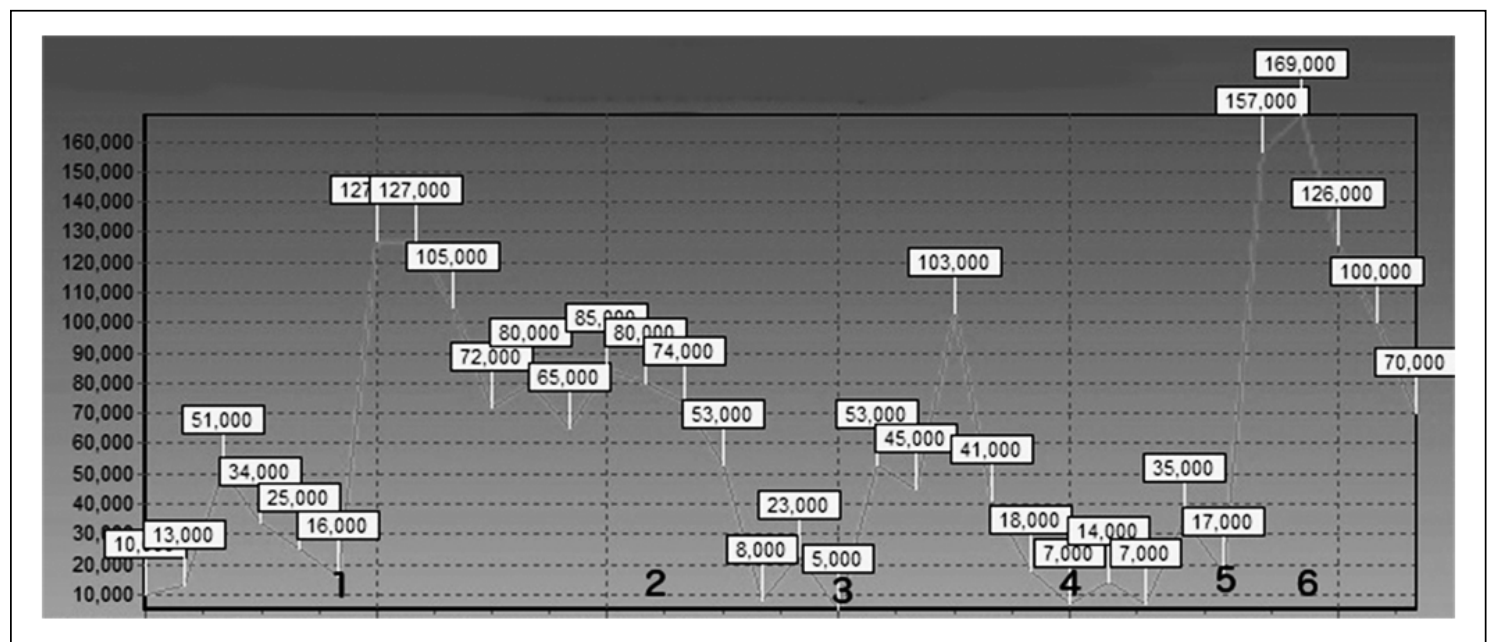

Figura 2. Control plaquetario del paciente. 1) Ingreso a cirugía urgente; 2) Inicio de glucocorticoides; 3) Primer dosis de inmunoglobulina; 4) Segunda dosis de inmunoglobulina; 5) Esplenectomía laparoscópica; 6) Deterioro neurológico, con hematoma subdural e isquemia hemisférica derecha.

realizándose drenaje de quiste tumoral aspecto de hemangioblastoma, muy vascularizado, con resección macroscópica total (Figura 3A). El informe de patología fue compatible con hemangioblastoma. Dada la asociación con el síndrome de Von Hippel Lindau, se consideró adicionalmente búsqueda de otros tumores a nivel abdominal con ecografía la cual fue negativa.

Es trasladado a unidad de cuidado crítico con excelente respuesta neurológica sin resolución de trombocitopenia (Figura 2) -entre 5.000 y $169.000 / \mathrm{mL}-$ requiriendo en varias ocasiones so- porte transfusional buscando recuentos $>100.000 /$ $\mathrm{mL}$. El seguimiento tomográfico (Día 2 y 7 postoperatorio) demostraban hematoma subdural con signos de herniación en resolución. Es llevado a estudio de médula ósea (mielograma, biopsia, cariotipo) todos normales y con sospecha de púrpura trombócitopenica inmune (PTI) se indicó glucocorticoides (hidrocortisona $50 \mathrm{mg} \mathrm{c} / 6 \mathrm{~h}$ por 2 días, dexametasona $4 \mathrm{mg}$ c/ 6 h por 10 días) sin respuesta. En vista de riesgo de sangrado intracerebral se decidió aplicar inmunoglobulina IV $1 \mathrm{~g} / \mathrm{kg}$ por dos dosis espaciadas una semana más prednisona 


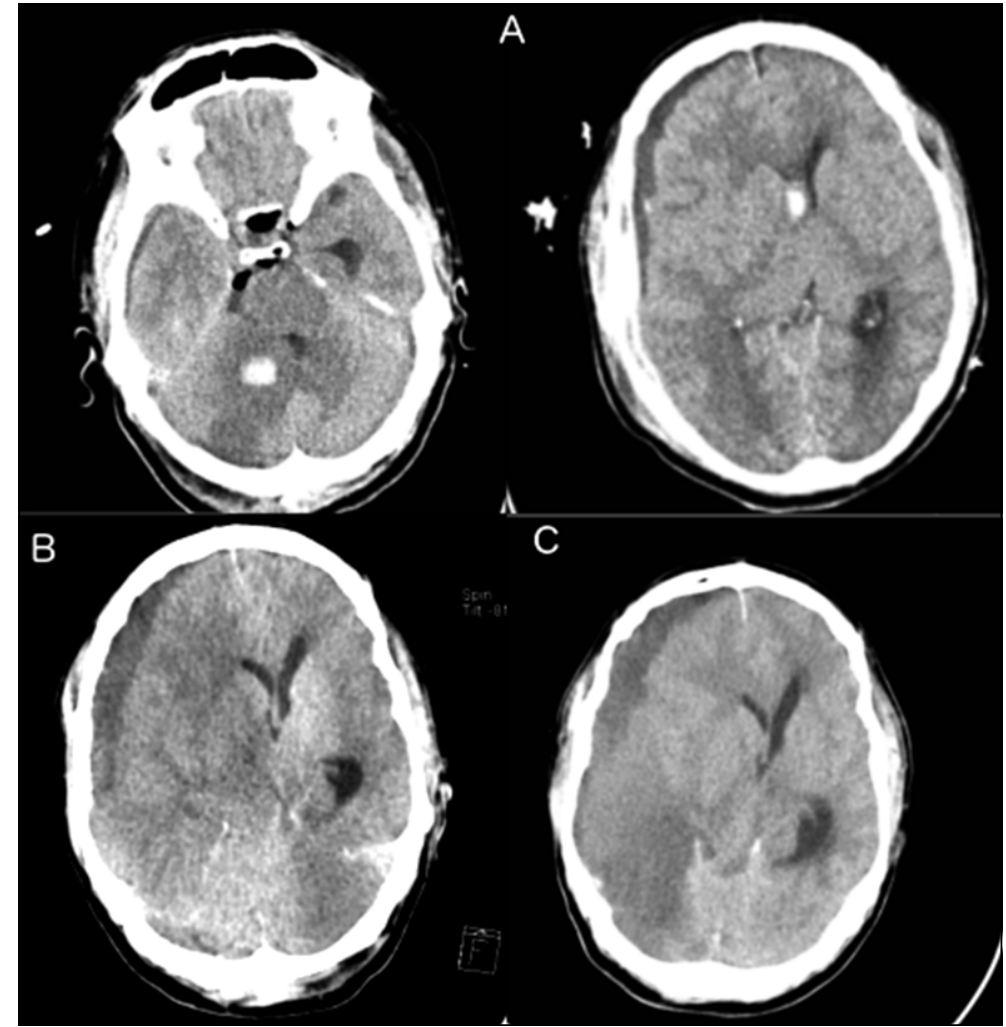

Figura 3. Controles tomográficos. A. Cortes tomográficos postoperatorio $24 \mathrm{~h}$, se observa pequeño sangrado en sitio previo del tumor y hematoma subdural pequeño con desviación de línea media; B. Tomografía $48 \mathrm{~h}$ postesplenectomía con hematoma subdural subagudo con efecto de masa y herniación subfalcina, hipodensidad de todo el hemisferio cerebral derecho; C. Control $72 \mathrm{H}$ postesplenectomía. Persistencia de hematoma sin cambios significativos, evento isquémico en evolución en territorio posterior derecho. a $1 \mathrm{mg} / \mathrm{kg} /$ día sin mejoría clínica por lo que se programó para esplenectomía laparoscópica 20 días después del procedimiento neuroquirúrgico. El procedimiento se desarrolla sin complicaciones y presenta recuento plaquetario de $165.000 / \mathrm{mL}$ en el postoperatorio inmediato, con bazo histológicamente normal. Cuarenta y ocho horas después, el paciente presenta nuevo deterioro neurológico requiriendo reingreso a UCI. Recuento plaquetario $126.000 / \mathrm{mL}$, tromboelastografía con MA elevado sugerente de hiperagregabilidad plaquetaria y TC de cráneo (Figura 3B) con hematoma subdural derecho subagudo con efecto de masa y tendencia a herniación con hipodensidad de todo el hemisferio cerebral ipsilateral sugestivo de isquemia por lo que se lleva nuevamente a cirugía de emergencia-drepanotomía-. El TC posterior (Figura 3C) no demostró cambios significativos. Con estos hallazgos se retira sedación del paciente, 2 días después presenta hipernatremia y fiebre, luego de dos evaluaciones se declara muerte encefálica.

\section{Discusión}

La cefalea es un motivo de consulta frecuente que rara vez se explica por tumores cerebrales. Signos de alarma como los encontrados en este paciente (cefalea gravativa, sin respuesta a analgésicos y con focalización neurológica) exigen la toma de neuroimágenes ${ }^{2}$.

Los hemangioblastomas -tumores benignos de bajo grado originados de capilares vascularespueden presentarse en cualquier sitio teniendo mayor preferencia por el cerebelo. Algunos están asociados a la enfermedad de von Hippel-Lin$\mathrm{dau}^{4}$ y el $\mathrm{KMS}^{5}$ pero la gran mayoría de ellos son esporádicos ${ }^{4}$.

El KMS se caracteriza por la presencia de tumores vasculares -principalmente hemangioendotelioma kaposiforme- $y$ trombocitopenia. Usualmente se acompaña de coagulopatía de consumo y anemia hemolitica microangiopática ${ }^{14}$. Este síndrome ha sido reportado con hemangio- 
mas hepáticos, esplénico, cerebeloso o del tejido celular subcutaneo ${ }^{15}$.

Yasui et $\mathrm{al}^{8}$ reportaron la serie más grande de KMS en Japón. En esta serie retrospectiva de 1999 a 2012 la edad media fue de 35 días, 55\% de sexo masculino con lesiones principalmente cervicofaciales y recuentos plaquetarios entre 3.000 y $76.000 / \mathrm{mL}$ (media 9.000). El diagnóstico se presenta en la niñez y muy infrecuentemente en el adulto lo que explica retrasos para el diagnóstico $y$ tratamiento adecuados en estos ${ }^{15-17}$.

El manejo del hemangioblastoma es netamente quirúrgico ${ }^{6}$ con el cual los niveles plaquetarios también responden. Antes de cirugía se puede usar esteroides a dosis equivalentes $2 \mathrm{mg} / \mathrm{kg} / \mathrm{día}$ de prednisona con lo que responden hasta $66 \%$ de los pacientes en sus recuentos plaquetarios ${ }^{7}$. Sin embargo, Yasui et $\mathrm{al}^{8}$ reportaron que $72 \%$ de los pacientes no presentaron respuesta a los esteroides y requirieron de otras opciones de tratamiento: vincristina ${ }^{4}$, interferon ${ }^{9,10}$ y radioterapia ${ }^{11}$.

La persistencia de trombocitopenia asociado al alto riesgo de sangrado en sistema nervioso central por la cirugía reciente no permitió el uso de otras terapias (radioterapia) concomitantes en este paciente. Bajo la sospecha de PTI se ofreció terapia de segunda (inmunoglobulina) y tercera línea (esplenectomía) con normalización de recuentos plaquetarios y posterior evento hemorrágico e isquémico. Los eventos isquémicos cerebrales han sido reportados como complicación de esplenectomía por trombocitosis secundaria ${ }^{12} y$ explican muy posiblemente el descenlace fatal de este paciente. En nuestro paciente no se desarrolló trombocitosis, pero en el tromboelastograma se identificó un aumento de la amplitud máxima (MA) como manifestación de aumento entre la relación de la fibrina y las plaquetas, aunque el ángulo no estaba aumentado ${ }^{13}$.

Aunque este paciente experimentó resección completa de la lesión, su recuento plaquetario no mejoró. El hemangioblastoma podría predisponer al desarrollo de autoanticuerpos plaquetarios y desencadenar PTI, mecanismo similar al reportado con otro tumor vascular en la literatura ${ }^{18}$. Otra posibilidad es la expresión de marcadores en el tumor que faciliten la agregación plaquetaria y su consumo como se ha descrito en el angiosarcoma $^{19}$.

El paciente no cursa con un KMS, única enfermedad descrita que asocia tumores vasculares y trombocitopenia por: la edad de presentación, niveles muy bajos de plaquetas sin respuesta a resección tumoral y el desarrollo de posible complicación (evento isquémico cerebral) asociada a la esplenectomía tampoco favorece el diagnóstico. El VHL es poco probable en vista de la edad de presentación y la ausencia de lesiones retinianas y abdominales en la tamización inicial. No fue posible medición de VHL dada la preocupación por la persistencia de trombocitopenia y complicaciones neurológicas asociadas.

En la literatura, sólo existen 2 reportes similares, ambos en francés y en los sesentas ${ }^{17}$. Se requerirá de un mayor interés en la literatura para definir si se trata de un nuevo síndrome en medicina. De los reportes previos no se puede determinar su histología comparada con el caso actual y podrían tratarse de hemangiomas. Como diagnóstico diferencial tendríamos infarto cerebral secundario a hematoma subdural crónico agudizado, sin embargo, esta complicación es más frecuente en niños y sólo se presenta en $0,4 \%$ de los casos luego de neurocirugía ${ }^{20}$.

Se presenta un caso en el adulto de asociación entre hemangioblastoma cerebeloso y trombocitopenia que dificultó el manejo inicial, sin respuesta al tratamiento instaurado y desenlace fatal.

\section{Referencias}

1. Jensen R, Stovner LJ. Epidemiology and comorbidity of headache. Lancet Neurol 2008; 7 (4): 354-61.

2. Hainer BL, Matheson EM. Approach to acute headache in adults. Am Fam Physician 2013; 87 (10): 682-7.

3. Kirby S, Purdy RA. Headaches and Brain Tumors. Neurol Clin 2014; 32 (2): 423-32.

4. Liao C-C, Huang Y-H. Clinical features and surgical outcomes of sporadic cerebellar hemangioblastomas. Clin Neurol Neurosurg 2014; 125: 160-5.

5. Nakamura, Ohnishi, Watanabe, Takashi. Correspondence. Br J Dermatol 1998; 139 (1): 164-6.

6. Hall GW. Kasabach-Merritt syndrome: pathogenesis and management. Br J Haematol 2001; 112 (4): 851-62.

7. Abass K, Saad H, Kherala M, Abd-Elsayed AA. Successful treatment of kasabach-merritt syndrome with vincristine and surgery: a case report and review of literature. Cases J 2008; 1 (1): 9.

8. Yasui N, Koh K, Kato M, Park M, Tomizawa D, Oshima $\mathrm{K}$, et al. Kasabach-Merritt phenomenon: a report of 11 cases from a single institution. J Pediatr Hematol Oncol 2013; 35 (7): 554-8. 
9. Acharya S, Pillai K, Francis A, Criton S, Parvathi VK. Kasabach merritt syndrome: management with interferon. Indian J Dermatol 2010; 55 (3): 281-3.

10. Akyüz C, Emir S, Büyükpamukçu M, Büyükpamukçu N, Cağlar M, Kale G, et al. Successful treatment with interferon alfa in infiltrating angiolipoma: a case presenting with Kasabach-Merritt syndrome. Arch Dis Child 2003; 88 (1): 67-8.

11. Mitsuhashi N, Furuta M, Sakurai H, Takahashi T, Kato $\mathrm{S}$, Nozaki M, et al. Outcome of radiation therapy for patients with Kasabach-Merritt syndrome. Int J Radiat Oncol Biol Phys 1997; 39 (2): 467-73.

12. Kim B-J, Park K-W, Koh S-B, Kim H-K, Jung HL, Park $\mathrm{M}-\mathrm{K}$, et al. Stroke induced by splenectomy in hemoglobin Madrid: autopsy clues to the underlying mechanism. Blood Coagul Fibrinolysis Int J Haemost Thromb 2005;16 (2): 141-4.

13. Raffán Sanabria F, P R, J F, Cuervo JA, Marín S, F L. Tromboelastografía. Rev Colomb Anestesiol 2005; 33 (3):181-6.

14. Sridhar S, Kuruvilla KA. Kasabach-Merritt syndrome.
Indian Pediatr 2005; 42 (10): 1045-6.

15. Mizuno J, Nakagawa H, Watabe T, Sugimoto I. Spontaneous spinal epidural haematoma in association with Kasabach-Merritt syndrome. J Clin Neurosci Off J Neurosurg Soc Australas 1999; 6 (6): 518-20.

16. Boros $\mathrm{G}$, Romhànyi $\mathrm{M}$, Horvàth $\mathrm{A}$, Tamàsi K. Kasabach-Merritt syndrome in an adult. Haematologia (Budap) 1973; 7 (1): 83-96.

17. Scherrer JR, Hausser E, Berney J. [Thrombocytopenia associated with a cerebellar hemangioblastoma]. Schweiz Med Wochenschr 1965; 95 (43): 1456-9.

18. Kitamura H, Ito S, Kuwana N, Yutani C. Epithelioid hemangioma of the temporal artery clinically mimicking temporal arteritis. Pathol Int 1999; 49(9): 831-5.

19. Satoh T, Takahashi Y, Yokozeki H, Katayama I, Nishioka K. Cutaneous angiosarcoma with thrombocytopenia. J Am Acad Dermatol 1999; 40 (5 Pt 2): 872-6.

20. Mori K, Maeda M. Surgical treatment of chronic subdural hematoma in 500 consecutive cases: clinical characteristics, surgical outcome, complications, and recurrence rate. Neurol Med Chir (Tokyo) 2001; 41 (8): 371-81. 